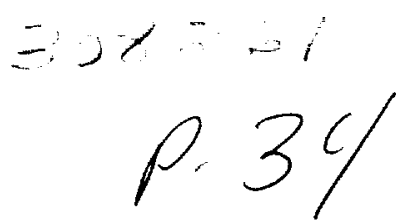

\title{
A NeW MOMENTUM MANAGEMENT CONTROLLER FOR THE SPACE STATION
}

\author{
B. Wie \\ K.W. Byun \\ V.W. Warren \\ University of Texas At Austin
}

January 1988

Cooperative Agreement NCC $9-16$

Research Activity No. MS.1

NASA Johnson Space Center

MIssion Support Directorate

Mission Planning and Analysis Division

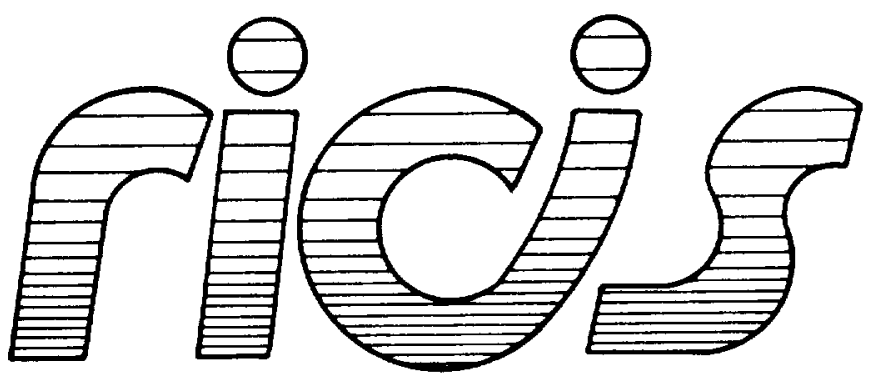

Research Institute for Computing and Information Systems University of Houston - Clear Lake

$$
T \cdot E \cdot C \cdot H \cdot N \cdot I \cdot C \cdot A \cdot L \quad R \cdot E \cdot P \cdot O \cdot R \cdot T
$$




\section{The RICIS Concept}

The University of Houston-Clear Lake established the Research Institute for Computing and Information systems in 1986 to encourage NASA Johnson Space Center and local industry to actively support research in the computing and information sciences. As part of this endeavor, UH-Clear Lake proposed a partnership with JSC to jointly define and manage an integrated program of research in advanced data processing technology needed for JSC's main missions, including administrative, engineering and science responsibilities. JSC agreed and entered into a three-year cooperative agreement with UH-Clear Lake beginning in May, 1986, to jointly plan and execute such research through RICIS. Additionally, under Cooperative Agreement NCC 9-16, computing and educational facilities are shared by the two institutions to conduct the research.

The mission of RICIS is to conduct, coordinate and disseminate research on computing and information systems among researchers, sponsors and users from UH-Clear Lake, NASA/JSC, and other research organizations. Within UH-Clear Lake, the mission is being implemented through interdisciplinary involvement of faculty and students from each of the four schools: Business, Education, Human Sciences and Humanities, and Natural and Applied Sciences.

Other research organizations are involved via the "gateway" concept. UH-Clear Lake establishes relationships with other universities and research organizations, having common research interests, to provide additional sources of expertise to conduct needed research.

A major role of RICIS is to find the best match of sponsors, researchers and research objectives to advance knowledge in the computing and information sciences. Working jointly with NASA/JSC, RICIS advises on research needs, recommends principals for conducting the research, provides technical and administrative support to coordinate the research, and integrates technical results into the cooperative goals of UH-Clear Lake and NASA/JSC. 


\section{A NeW MOMENTUM MANAGEMENT CONTROLLER FOR THE SPACE STATION}


$=$

$=$

$-$

- 


\section{Preface}

This research was conducted under the auspices of the Research Institute for Computing and Information Systems at the University of Houston-Clear Lake by Bong Wie, Assistant Professor, K.W. Byun and V.W. Warren, Graduate Research Assistants, all of the University of Texas at Austin. Terry Feagin, Professor of Computer Science, at the University of Houston-Clear Lake, served as technical representative for RICIS.

Funding has been provided by the Mission Planning and Analysis Division, within the Mission Support Directorate, NASA/JSC through Cooperative Agreement NCC 9-16 between NASA Johnson Space Center and the University of Houston-Clear Lake. The Technical Monitor for this activity was Aldo Bordano, Chief, Guidance and Navigation Branch, NASA/JSC.

The views and conclusions contained in this report are those of the authors and should not be interpreted as representative of the official policies, either express or impied, of NASA or the United States Government. 

submitted to the AIAA Journal of Guidance, Control, and Dynamics $(1 / 19 / 88)$

to be presented at NASA/JSC (2/4/88)

to be presented at the AIAA Guidance, Navigation, \& Control Conference

(August 1988)

\title{
A New Momentum Management Controller for the Space Station*
}

\author{
B. Wie, ${ }^{\dagger}$ K.W. Byun, ${ }^{\ddagger}$ and V.W. Warren ${ }^{\ddagger}$ \\ The University of Texas at Austin \\ Austin, Texas \\ D. Geller, ${ }^{\S}$ D. Long, ${ }^{\uparrow}$ and J. Sunkel ${ }^{\S}$ \\ NASA Johnson Space Center \\ Houston, Texas
}

\begin{abstract}
A new approach to CMG (control moment gyro) momentum management and attitude control of the space station is developed. The control algorithm utilizes both the gravity-gradient and gyroscopic torques to seek torque equilibrium attitude in the presence of secular and cyclic disturbances. We show that depending upon mission requirements, either pitch attitude or pitch-axis CMG momentum can be held constant. We also show that yaw attitude and roll-axis CMG momentum can be held constant while roll attitude and yaw-axis CMG momentum cannot be held constant. As a result, the overall attitude and CMG momentum oscillations caused by cyclic aerodynamic disturbances are minimized. A state feedback controller with minimal computer storage requirement for gain scheduling is also developed. The overall closed-loop system is stable for $\pm 30 \%$ inertia matrix variations and has more than $\pm 10 \mathrm{~dB}$ and $45^{\circ}$ stability margins in each loop.
\end{abstract}

"This work was supported by the NASA Johnson Space Center through the RICIS program of the University of Houston at Clear Lake.

'Assistant Professor, Dept. of Aerospace Engineering and Engineering Mechanics, Member AIA A.

'Graduate Research Assistants, Member AIAA.

Aerospace Engineers, Member AIAA.

Thead, Guidance Analysis Section, Mission Planning and Analysis Division. 


\section{Introduction}

The space station shown in Fig. 1 will employ CMGs (control moment gyros) as a primary actuating device during normal flight mode operation. Since the CMGs are momentum exchange devices, external control torques must be used to desaturate the CMGs, that is, bring the momentum back to nominal value. Some methods for unloading CMG momentum include the use of magnetic torques, reaction jets, and gravity-gradient torque. For the space station, the gravity-gradient torque approach is preferred since it requires no consumables or additional hardware. For this reason, various schemes using gravity-gradient torque have been developed [1-9].

Two approaches to momentum management using gravity-gradient torque are possible. The first of these is a "discrete" or "periodic" momentum control approach [1-5] which is basically a feedforward open-loop control scheme. This scheme utilizes appropriate samples of CMG momentum, with the sampling frequency of the same order as the orbital frequency. This approach, as well as the "predictive" approach [7], can handle expected momentum changes; however, it requires accurate inertia matrix properties and environmental models to generate the proper attitude steering command. In the "discrete" or "periodic" approach, the attitude controller, with a much higher control bandwidth, is designed independently.

The second approach to CMG momentum management is a "continuous" approach [6-9] which integrates the momentum management and attitude control design. In this continuous, closed-loop control of both the CMG momentum and station attitude, the design objective is to establish a proper tradeoff between station pointing and CMG momentum management, while satisfying the specific mission requirements.

Expanding on this "continuous" approach, we present a new scheme to the space station momentum management and attitude control. The proposed controller provides a proper disturbance accommodation. As a result, the cyclic peak of the station attitude and CMG momentum oscillation caused by aerodynamic torque is minimized. In this paper, CMGs are considered as an ideal torquer; however, the CMIG gimbal dynamics as well as the CMG steering law [10,11] should be included in the further development of an efficient control law for the overall system. Structural flexibility of the space station considered in $[12,13]$ is neglected because of the 
low bandwidth nature of the integrated momentum/attitude controller. We assume that a strapdown inertial reference system provides relatively noise-free estimates of all states and that the body-axis components of CMG momentum can be measured. Practical multivariable controller synthesis is accomplished employing various techniques such as the classical control approaches [12-1 $\bar{\tau}]$, linear-quadratic-regulator (LQR) synthesis technique $[18,19]$, asymptotic disturbance rejection $[19,20]$, decentralized (partial state feedback) control [21], and robust eigensystem assignment techniques [22-24]. A brief summary of a pole placement algorithm for partial state feedback controller is given in Appendix.

\section{Mathematical Models for Controller Synthesis}

The space station in circular orbit is expected to maintain LVLH (local vertical and local horizontal) orientation during normal mode operation. The nonlinear equations of motion in terms of components along the body-fixed control axes can be written as:

Space Station Dynamics:

$$
\begin{gathered}
{\left[\begin{array}{lll}
I_{11} & I_{12} & I_{13} \\
I_{21} & I_{22} & I_{23} \\
I_{31} & I_{32} & I_{33}
\end{array}\right]\left[\begin{array}{l}
\dot{\omega}_{1} \\
\dot{\omega}_{2} \\
\dot{\omega}_{3}
\end{array}\right]=-\left[\begin{array}{ccc}
0 & -\omega_{3} & \omega_{2} \\
\omega_{3} & 0 & -\omega_{1} \\
-\omega_{2} & \omega_{1} & 0
\end{array}\right]\left[\begin{array}{lll}
I_{11} & I_{12} & I_{13} \\
I_{21} & I_{22} & I_{23} \\
I_{31} & I_{32} & I_{33}
\end{array}\right]\left[\begin{array}{l}
\omega_{1} \\
\omega_{2} \\
\omega_{3}
\end{array}\right]} \\
+3 n^{2}\left[\begin{array}{ccc}
0 & -c_{3} & c_{2} \\
c_{3} & 0 & -c_{1} \\
-c_{2} & c_{1} & 0
\end{array}\right]\left[\begin{array}{lll}
I_{11} & I_{12} & I_{13} \\
I_{21} & I_{22} & I_{23} \\
I_{31} & I_{32} & I_{33}
\end{array}\right]\left[\begin{array}{l}
c_{1} \\
c_{2} \\
c_{3}
\end{array}\right]+\left[\begin{array}{l}
-u_{1}+w_{1} \\
-u_{2}+w_{2} \\
-u_{3}+w_{3}
\end{array}\right]
\end{gathered}
$$

where

$$
\begin{aligned}
& c_{1} \triangleq-\sin \theta_{2} \cos \theta_{3} \\
& c_{2} \triangleq \cos \theta_{1} \sin \theta_{2} \sin \theta_{3}+\sin \theta_{1} \cos \theta_{2} \\
& c_{3} \triangleq-\sin \theta_{1} \sin \theta_{2} \sin \theta_{3}+\cos \theta_{1} \cos \theta_{2}
\end{aligned}
$$

Attitude Kinematics (2-3-1 body-axis sequence):

$$
\left[\begin{array}{l}
\dot{\theta}_{1} \\
\dot{\theta}_{2} \\
\dot{\theta}_{3}
\end{array}\right]=\frac{1}{\cos \theta_{3}}\left[\begin{array}{rrr}
\cos \theta_{3}, & -\cos \theta_{1} \sin \theta_{3}, & \sin \theta_{1} \sin \theta_{3} \\
0, & \cos \theta_{1}, & -\sin \theta_{1} \\
0, & \sin \theta_{1} \cos \theta_{3}, & \cos \theta_{1} \cos \theta_{3}
\end{array}\right]\left[\begin{array}{l}
\omega_{1} \\
\omega_{2} \\
\omega_{3}
\end{array}\right]+\left[\begin{array}{l}
0 \\
n \\
0
\end{array}\right]
$$


CMG Momentum:

$$
\left[\begin{array}{l}
\dot{h}_{1} \\
\dot{h}_{2} \\
\dot{h}_{3}
\end{array}\right]+\left[\begin{array}{ccc}
0 & -\omega_{3} & \omega_{2} \\
\omega_{3} & 0 & -\omega_{1} \\
-\omega_{2} & \omega_{1} & 0
\end{array}\right]\left[\begin{array}{l}
h_{1} \\
h_{2} \\
h_{3}
\end{array}\right]=\left[\begin{array}{l}
u_{1} \\
u_{2} \\
u_{3}
\end{array}\right]
$$

where $(1,2,3)$ are the roll, pitch, and yaw control axes whose origin is fixed at the mass center, with the roll axis in the flight direction, the pitch axis perpendicular to the orbit plane, and the yaw axis toward the Earth; $\left(\theta_{1}, \theta_{2}, \theta_{3}\right)$ are the roll, pitch, yaw Euler angles of the control (body) axes with respect to LVLH axes which rotate with the orbital angular velocity, $n ;\left(\omega_{1}, \omega_{2}, \omega_{3}\right)$ are the body-axis components of the absolute angular velocity of the station; $\left(I_{11}, I_{22}, I_{33}\right)$ are the moments of inertia; $I_{i j}$ $(i \neq j)$ are the products of inertia; $\left(h_{1}, h_{2}, h_{3}\right)$ are the body-axis components of the CMG momentum; $\left(u_{1}, u_{2}, u_{3}\right)$ are the body-axis components of the control torque caused by CMG momentum change; $\left(w_{1}, w_{2}, w_{3}\right)$ are the body-axis components of the external disturbance torque; and $n$ is the orbital rate of $0.0011 \mathrm{rad} / \mathrm{sec}$.

For small attitude deviations from LVLH orientation, the linearized equations of motion can be written as:

Space Station Dynamics:

$$
\begin{aligned}
& {\left[\begin{array}{lll}
I_{11} & I_{12} & I_{13} \\
I_{21} & I_{22} & I_{23} \\
I_{31} & I_{32} & I_{33}
\end{array}\right]\left[\begin{array}{l}
\dot{\omega}_{1} \\
\dot{\omega}_{2} \\
\dot{\omega}_{3}
\end{array}\right]=n\left[\begin{array}{rrr}
I_{31}, & 2 I_{32,} & I_{33}-I_{22} \\
-I_{32}, & 0, & I_{12} \\
I_{22}-I_{11}, & -2 I_{12}, & -I_{13}
\end{array}\right]\left[\begin{array}{l}
\omega_{1} \\
\omega_{2} \\
\omega_{3}
\end{array}\right]} \\
& +3 n^{2}\left[\begin{array}{crr}
I_{33}-I_{22}, & I_{21}, & 0 \\
I_{12}, & I_{33}-I_{11}, & 0 \\
-I_{13}, & -I_{23}, & 0
\end{array}\right]\left[\begin{array}{l}
\theta_{1} \\
\theta_{2} \\
\theta_{3}
\end{array}\right]+n^{2}\left[\begin{array}{r}
-2 I_{23} \\
3 I_{13} \\
-I_{12}
\end{array}\right]+\left[\begin{array}{c}
-u_{1}+\mathrm{w}_{1} \\
-u_{2}+\mathrm{w}_{2} \\
-u_{3}+\mathrm{w}_{3}
\end{array}\right]
\end{aligned}
$$

Attitude Kinematics:

$$
\begin{aligned}
& \dot{\theta}_{1}-n \theta_{3}=\omega_{1} \\
& \dot{\theta}_{2}-n=\omega_{2} \\
& \dot{\theta}_{3}+n \theta_{1}=\omega_{3}
\end{aligned}
$$

CMG Momentum:

$$
\begin{aligned}
& \dot{h}_{1}-n h_{3}=u_{1} \\
& \dot{h}_{2}=u_{2} \\
& \dot{h}_{3}+n h_{1}=u_{3}
\end{aligned}
$$


Eliminating $\left(\omega_{1}, \omega_{2}, \omega_{3}\right)$ from Eqs. 4 and 5 , the linearized equations of motion can also be written as:

$$
\begin{gathered}
{\left[\begin{array}{lll}
I_{11} & I_{12} & I_{13} \\
I_{21} & I_{22} & I_{23} \\
I_{31} & I_{32} & I_{33}
\end{array}\right]\left[\begin{array}{l}
\ddot{\theta}_{1} \\
\ddot{\theta}_{2} \\
\ddot{\theta}_{3}
\end{array}\right]=n\left[\begin{array}{rrr}
0, & 2 I_{32}, & I_{11}-I_{22}+I_{33} \\
-2 I_{32}, & 0, & 2 I_{12} \\
-I_{11}+I_{22}-I_{33}, & -2 I_{12}, & 0
\end{array}\right]\left[\begin{array}{l}
\dot{\theta}_{1} \\
\dot{\theta}_{2} \\
\dot{\theta}_{3}
\end{array}\right]} \\
\left.+n^{2}\left[\begin{array}{ccc}
4\left(I_{33}-I_{22}\right), & 3 I_{21}, & -I_{31} \\
4 I_{12}, & 3\left(I_{33}-I_{11}\right), & I_{32} \\
-4 I_{13}, & -3 I_{23}, & I_{11}-I_{22}
\end{array}\right]\left[\begin{array}{l}
\theta_{1} \\
\theta_{2} \\
\theta_{3}
\end{array}\right]+n^{2} \cdot \begin{array}{r}
-4 I_{23} \\
3 I_{13} \\
I_{12}
\end{array}\right]+\left[\begin{array}{r}
-u_{1}+\mathrm{w}_{1} \\
-u_{2}+\mathrm{w}_{2} \\
-u_{3}+\mathrm{w}_{3}
\end{array}\right](7)
\end{gathered}
$$

Note that the products of inertia cause three-axis coupling as well as a bias torque in each axis. Early flight configurations during station assembly may have significant misalignment of the control axes with the principal axes. In such cases, the above linear, 3-axis coupled equations of motion will be very useful for linear control design. It is by now evident that it is very straightforward to derive Eq. 7; however, this does not appear to have been done in the literature to the authors' knowledge.

Fortunately, most practical situations of interest with small products of inertia permit further simplication in such a way that pitch motion is uncoupled from roll/yaw motion. Otherwise, Eq. 7 should be used for 3-axis coupled stability analysis and control design. For the case where the control axes are nearly aligned with the principal axes $\left(I_{1} \triangleq I_{11}, I_{2} \triangleq I_{22}\right.$, and $\left.I_{3} \triangleq I_{33}\right)$, Eqs. 4 become

$$
\begin{aligned}
& I_{1} \dot{\omega}_{1}+n\left(I_{2}-I_{3}\right) \omega_{3}+3 n^{2}\left(I_{2}-I_{3}\right) \theta_{1}=-u_{1}+\mathrm{w}_{1} \\
& I_{2} \dot{\omega}_{2}+3 n^{2}\left(I_{1}-I_{3}\right) \theta_{2}=-u_{2}+\mathrm{w}_{2} \\
& I_{3} \dot{\omega}_{3}-n\left(I_{2}-I_{1}\right) \omega_{1}=-u_{3}+\mathrm{w}_{3}
\end{aligned}
$$

Finally, the linear equations for $\theta_{1}, \theta_{2}$, and $\theta_{3}$ become

$$
\begin{aligned}
& I_{1} \ddot{\theta}_{1}+4 n^{2}\left(I_{2}-I_{3}\right) \theta_{1}-n\left(I_{1}-I_{2}+I_{3}\right) \dot{\theta}_{3}=-u_{1}+w_{1} \\
& I_{2} \ddot{\theta}_{2}+3 n^{2}\left(I_{1}-I_{3}\right) \theta_{2}=-u_{2}+w_{2} \\
& I_{3} \ddot{\theta}_{3}+n^{2}\left(I_{2}-I_{1}\right) \theta_{3}+n\left(I_{1}-I_{2}+I_{3}\right) \dot{\theta}_{1}=-u_{3}+w_{3}
\end{aligned}
$$

These are the important equations used for the study of passive or active gravitygradient stabilization of Earth-pointing satellites (e.g., see $[18,16])$. Since pitch motion is uncoupled from roll/yaw motion, pitch control is often treated separately from coupled roll/yaw control. 
However, certain configurations of the space station (e.g., the assembly flight \#3 of Table 1) may need a large pitch TEA because of the small gravity-gradient torque available in the pitch axis. In such cases, Eqs. 1 and 2 with small roll/yaw attitude errors and small products of inertia become $[25,26]$ :

$$
\begin{aligned}
& I_{1} \ddot{\theta}_{1}+\left(1+3 \cos ^{2} \theta_{2}\right) n^{2}\left(I_{2}-I_{3}\right) \theta_{1}-n\left(I_{1}-I_{2}+I_{3}\right) \dot{\theta}_{3} \\
& +3\left(I_{2}-I_{3}\right) n^{2}\left(\sin \theta_{2} \cos \theta_{2}\right) \theta_{3}=-u_{1}+\mathrm{w}_{1} \\
& I_{2} \ddot{\theta}_{2}+3 n^{2}\left(I_{1}-I_{3}\right) \sin \theta_{2} \cos \theta_{2}=-u_{2}+\mathrm{w}_{2} \\
& I_{3} \ddot{\theta}_{3}+\left(1+3 \sin ^{2} \theta_{2}\right) n^{2}\left(I_{2}-I_{1}\right) \theta_{3}+n\left(I_{1}-I_{2}+I_{3}\right) \dot{\theta}_{1} \\
& +3\left(I_{2}-I_{1}\right) n^{2}\left(\sin \theta_{2} \cos \theta_{2}\right) \theta_{1}=-u_{3}+\mathrm{w}_{3}
\end{aligned}
$$

It is evident that roll/yaw motion is now affected by pitch motion. If pitch attitude is held constant with respect to the pitch TEA, the roll/yaw equations can be considered as time invariant. Thus, these equations will also be useful for roll/yaw controller design for the space station with large pitch TEA.

Inertia matrices of the Phase I space station as well as the assembly flight \#3 are listed in Table 1 . In this paper we are concerned primarily with the phase 1 configuration. The uncontrolled space station with such inertial properties is in an unstable equilibrium when $\theta_{i}=0(i=1,2,3)$. Also included are expected aerodynamic disturbances which are modeled as bias plus cyclic terms in the bodyfixed control axes:

$$
\mathrm{w}(t)=\operatorname{Bias}+A_{n} \sin \left(n t+\phi_{n}\right)+A_{2 n} \sin \left(2 n t+\phi_{2 n}\right)
$$

The cyclic component at orbital rate is due to the diurnal bulge effect, while the cyclic torque at twice the orbital rate is caused by the rotating solar panels. The magnitudes and phases of aerodynamic torque in each axis are assumed unknown for control design.

\section{Pitch-Axis Controller Synthesis}

Equation $9 \mathrm{~b}$, which is linearized and uncoupled from the roll/yaw equations, is used as the basis for pitch control analysis and design. A block diagram of the pitchaxis momentum/attitude control loop is shown in Fig. 2. Two control schemes are 
presented here: one without the indicated cyclic-disturbance rejection filter. $C_{2}(s)$, and one including the filter.

\section{Pitch Control without Cyclic-Disturbance Rejection}

Pitch-axis controller consists of a single control input, $u_{2}$ and four states, $\theta_{2}, \dot{\theta}_{2}$, $h_{2}, \int h_{2}$. The pitch control logic is then given by

$$
u_{2}=K_{2 P} \theta_{2}+K_{2 D} \dot{\theta}_{2}+K_{2 H} h_{2}+\dot{K}_{2 I} \int h_{2}
$$

where the pitch-axis CMG momentum and its integral are included to prevent momentum build-up.

Various methods for the selection of the four gains can be employed. For example, one can place the closed-loop eigenvalues at any desired location, using the pole placement technique. The practical problem with this approach is that it is not always clear where to place the eigenvalues for satisfactory performance and robustness.

Pitch controller synthesis by iterative successive loop closures is possible, but quite tedious. The pitch controller can also be synthesized using an "equivalent" compensator. This approach provides physical insight into the proper tradeoff between the attitude control and the momentum management. Combining Eqs. $6 \mathrm{~b}$ and 12 to eliminate $h_{2}$ gives an equivalent compensator

$$
u_{2}=\frac{s^{2}\left(K_{2 P}+s K_{2 D}\right)}{s^{2}-K_{2 H} s-K_{2 I}} \theta_{2}
$$

Hence, the integrated momentum/attitude controller can be interpreted as a secondorder compensator with four parameters. The momentum controller consists of double zero at the origin and complex poles. The attitude controller consists of one zero on the real axis, which is a conventional proportional-derivative controller. An unstable compensator is needed to stabilize the unstable system. It can be easily shown that $h_{2}(s) / w_{2}(s)$ of the closed-loop system has a zero at $s=0$. Thus, $h_{2}(t)$ has a zero steady-state value for a constant disturbance.

LQR synthesis of the pitch control for feeding back all four states to $u_{2}$ can be done quickly using a computer code. The LQR technique makes use of a quadratic performance index to synthesize state-feedback gains that minimize this index. It is especially useful for multivariable systems; all loops are closed simultaneously 
instead of successively as in classical frequency-domain methods. However. the problem with this approach is that the proper selection of the weighting matrices is not obvious. It is not always possible to predict the effects of given weighting matrices on the closed-loop behavior.

Hence, a practical approach to the pitch-axis controller design (and the roll/yaw controller design of the next section) would be to find the control gains and closedloop poles that result from a wide range of weighting matrices, and simulate the corresponding closed-loop system. The gain matrix that produces the closed-loop responses satisfying the various requirements becomes the final selection.

The uncontrolled phase 1 space station has unstable pitch mode with poles at $s=$ $\pm 1.5 n$ and momentum mode with double pole at $s=0$ (one from integral feedback). After iterative use of an LQR synthesis code available in the PC-MATLAB and CTRL-C software, we select the pitch-axis control gains as listed in Table 2. The corresponding closed-loop eigenvalues are:

$$
-1.6 n, \quad-1.0 n, \quad(-1.5 \pm 1.5 j) n
$$

The response of the closed-loop system to the pitch disturbance listed in Table 1 , with initial conditions of $\theta_{2}(0)=1 \mathrm{deg}$ and $\dot{\theta}_{2}(0)=0.001 \mathrm{deg} / \mathrm{sec}$, are shown in Fig. 3. The transient responses are satisfactory, while the cyclic aerodynamic torque causes the periodic responses of both pitch attitude and pitch-axis CMG momentum. The $h_{2}$ is bounded with zero mean value, while the $\theta_{2}$ is oscillating with respect to $7.5^{\circ}$ pitch TEA. The CMG momentum peak and torque demand are both well below the allowable limits of about $20,000 \mathrm{ft}-\mathrm{lb}-\mathrm{sec}$ and $150 \mathrm{ft}-\mathrm{lb}$, respectively.

\section{Pitch Control with Cyclic-Disturbance Rejection}

Depending on the circumstances, either pitch attitude or CMG momentum oscillation, caused by the aerodynamic disturbance torque, may be undesirable. In such cases, a cyclic-disturbance rejection filter can be employed as illustrated in Fig. 2.

The proposed cyclic-disturbance rejection filter, $C_{2}(s)$, is represented as

$$
C_{2}(s)=\frac{N_{2}(s)}{\left[s^{2}+n^{2}\right]\left[s^{2}+(2 n)^{2}\right]}
$$


The filter poles will appear in the numerator of the closed-loop transfer function $\theta_{2}(s) / \mathrm{w}_{2}(s)$ or $h_{2}(s) / \mathrm{w}_{2}(s)$, depending on mode selection. This results in a disturbance rejection at frequencies of $n$ and $2 n$ for either $\theta_{2}$ or $h_{2}$. The filter numerator. $N_{2}(s)$, should be properly designed to stabilize the overall control system.

For example, the disturbance rejection filter for $\theta_{2}$ can be represented as

$$
\begin{aligned}
& \ddot{\alpha}_{2}+(n)^{2} \alpha_{2}=\theta_{2} \\
& \ddot{\beta}_{2}+(2 n)^{2} \beta_{2}=\theta_{2}
\end{aligned}
$$

where initial conditions for $\alpha_{2}, \beta_{2}, \dot{\alpha}_{2}$, and $\dot{\beta}_{2}$ can be arbitrarily selected (usually. zero initial conditions). These filter equations are integrated in the on-board computer.

We now repeat the pitch control synthesis of the previous section, augmenting the pitch-axis equation with the above filter equations. The pitch control logic, with additional disturbance rejection filter states, can then be expressed as

$$
u_{2}=K_{2 P} \theta_{2}+K_{2 D} \dot{\theta}_{2}+K_{2 H} h_{2}+K_{2 I} \int h_{2}+K_{2 \alpha} \alpha_{2}+K_{2 \dot{\alpha}} \dot{\alpha}_{2}+K_{2 \beta} \beta_{2}+K_{2 \dot{\beta}} \dot{\beta}_{2}
$$

The eight gains of Eq. 16 can be determined using either the pole placement or LQR technique. We use the LQR synthesis technique, and the selected control gains and the corresponding closed-loop eigenvalues are listed in Tables 3 and 4, respectively.

Since the disturbance rejection filter is used for pitch attitude, it can be shown that $\theta_{2}(s) / \mathrm{w}_{2}(s)$ has zeros at $s= \pm n j$ and $s= \pm 2 n j$; hence, an asymptotic disturbance rejection of a cyclic disturbance of frequencies $n$ and $2 n$ is achieved for the pitch attitude. Transfer function $h_{2}(s) / \mathrm{w}_{2}(s)$ also has zeros near $s= \pm n j$ and $\pm 2 n j$. This results in minimizing the cyclic peak of the CMG momentum. The closed-loop response of this controller is shown in Fig. 4. The CMG momentum is bounded with zero mean value, while the pitch attitude is now held constant at $7.5^{\circ}$ pitch TEA after two orbits. These responses should be compared to those in Fig. 3 where no disturbance rejection filter was used. It can be seen that the cyclic peak of the CMG momentum is also reduced. In other words, the use of disturbance rejection filtering results in minimizing the overall oscillations of attitude and CMG momentum.

It is also possible to provide cyclic-disturbance rejection for the pitch-axis CMG momentum. In this case, we replace $\theta_{2}$ of Eqs. 15 by $h_{2}$ and then find proper gains of 
Eq. 16. The closed-loop response for this case is shown in Fig. $\overline{5}$; hence, depending on the specific mission requirements, either pitch attitude or CMG momentum can be held constant employing the pitch controller illustrated in Fig. 2.

\section{Roll/Yaw Controller Synthesis}

A design procedure similar to that of the pitch-axis design is followed for the roll/yaw controller. Two cases are considered: the first without cyclic-disturbance rejection filters, the second with filters.

\section{Roll/Yaw Control without Cyclic-Disturbance Rejection}

The first case of roll/yaw controller design consists of two inputs, $u_{1}$ and $u_{3}$, and eight states, including two integral states for the CMG momentum. The eight states are:

$$
\begin{array}{lllll}
\text { Roll-axis: } & \theta_{1} & \omega_{1} & h_{1} & \int h_{1} \\
\text { Yaw-axis: } & \theta_{3} & \omega_{3} & h_{3} & \int h_{3}
\end{array}
$$

Note that we use $\omega_{1}$ and $\omega_{3}$, instead of $\dot{\theta}_{1}$ and $\dot{\theta}_{3}$, as state variables. The uncontrolled phase 1 space station with inertial property of Table 1 is unstable in roll/yaw and has the open-loop poles of $( \pm 1.05 \pm 0.7 j) n, \pm n j, 0,0$. The double pole at $s=0$ are due to the integral feedback of $h_{1}$ and $h_{3}$. Similar to the pitch-axis design, the roll/yaw full-state feedback controller can be designed, iteratively, using the LQR or pole placement techniques. The multi-input characteristics of the roll/yaw axes, however, provide for the calculation of various gain matrices which yield the same closed-loop eigenvalues. Although the gain matrix can be completely specified by assigning not only the closed-loop eigenvalues but also an allowable set of closed-loop eigenvectors, there are many open issues in the eigensystem assignment approach [22-24]. In our study, we simply use the conventional LQR technique to find a closed-loop system satisfying various requirements.

After iterative use of an LQR synthesis code, we select a $2 \times 8$ gain matrix with the corresponding closed-loop eigenvalues of $(-1.05 \pm 0.68 j) n,(-1.04 \pm 0.72 j) n$, $(-1.42 \pm 1.38 j) n$, and $(-1.42 \pm 1.38 j) n$. This choice of closed-loop eigenvalues is not claimed to be the best. 
We now discuss some motivation for developing a decentralized, partial state feedback controller for the space station. The configurations of the space station will be changing as payloads/modules are attached or removed. This drives the need for a control system design that is either insensitive to the configuration changes or able to adapt autonomously. At present, conventional design methods. including gain scheduling or mode switching, appear adequate for most of the space station flight configurations. Some drawbacks of non-adaptive controllers are that the design is often time-consuming, and the controller parameters (e.g., the 16 gains of the above roll/yaw controller) must be determined for many operating conditions. Furthermore, they must be stored in the on-board computer.

In order to minimize the computer storage requirement for gain scheduling, a roll/yaw control logic with reduced number of gains is considered here. One possibility might be to use an LQR synthesis code to find a full-state feedback gain matrix and then omit some of the less important gains. However, this approach does not always guarantee good performance and stability. Hence, we have developed an iterative pole placement algorithm which is an extension of the Bass-Gura formula for single-input systems. A brief summary of the algorithm for multi-input systems is given in Appendix.

A decentralized, partial state feedback control logic proposed for the space station is simply:

$$
\begin{aligned}
& u_{1}=K_{1 P} \theta_{1}+K_{1 D} \omega_{1}+K_{1 H} h_{1}+K_{1 I} \int h_{1} \\
& u_{3}=K_{3 P} \theta_{3}+K_{3 D} \omega_{3}+K_{3 H} h_{3}+K_{3 I} \int h_{3}
\end{aligned}
$$

Notice a particular feedback structure where each control input uses states which are more closely related to that control input. We select the eight gains by choosing the closed-loop eigenvalues to be the optimal, full-state regulator eigenvalues determined previously. The eight gains obtained using the pole placement algorithm described in Appendix are listed in Table 2. A simulation of the closed-loop system with roll/yaw disturbances of Table 1 shows that the transient responses are satisfactory and that the steady-state responses are cyclic and remain within specified limits. The decentralized controller with eight gains compares quite favorably with the full-state feedback controller with sixteen gains. 


\section{Roll/Yaw Control with Cyclic-Disturbance Rejection}

For the minimization of the steady-state oscillation of roll/yaw attitude and CMG momentum, cyclic-disturbance rejection filtcring for a multirariable system is investigated.

To clarify some multivariable control issues related to transmission zeros [20]. let us consider the open-loop transfer function matrix from control inputs to roll/yaw attitude and CMG momentum. Combining Eqs. 6a. 6c, 9a, and 9c. we can find the transfer function matrix as:

$$
\begin{aligned}
{\left[\begin{array}{l}
\theta_{1}(s) \\
\theta_{3}(s)
\end{array}\right]=} & \frac{1}{\Delta}\left[\begin{array}{rr}
-\left[I_{3} s^{2}+\left(I_{2}-I_{1}\right) n^{2}\right]\left(s^{2}+n^{2}\right), & -\left[\left(I_{1}-I_{2}+I_{3}\right) n s\right]\left(s^{2}+n^{2}\right) \\
{\left[\left(I_{1}-I_{2}+I_{3}\right) n s\right]\left(s^{2}+n^{2}\right),} & -\left[I_{1} s^{2}+4\left(I_{2}-I_{3}\right) n^{2}\right]\left(s^{2}+n^{2}\right)
\end{array}\right] \\
& {\left[\begin{array}{r}
u_{1}(s)-\mathrm{w}_{1}(s) \\
u_{3}(s)-\mathrm{w}_{3}(s)
\end{array}\right] } \\
{\left[\begin{array}{l}
h_{1}(s) \\
h_{3}(s)
\end{array}\right]=} & \frac{1}{s^{2}+n^{2}}\left[\begin{array}{rr}
s, & n \\
-n, & s
\end{array}\right]\left[\begin{array}{l}
u_{1}(s) \\
u_{3}(s)
\end{array}\right]
\end{aligned}
$$

where $\Delta=I_{1} I_{3}\left(s^{2}+n^{2}\right)\left[s^{4}+\left(1+3 k_{1}+k_{1} k_{3}\right) n^{2} s^{2}+4 k_{1} k_{3} n^{4}\right], k_{1}=\left(I_{2}-I_{3}\right) / I_{1}, k_{3}=$ $\left(I_{2}-I_{1}\right) / I_{3}$.

It is apparent that $\pm n j$ are transmission zeros of Eq. 18. We also notice from Eq. 19 that resonance of CMG momentum can happen for sinusoidal control inputs of frequency $n$. In other words, a cyclic-disturbance at orbital rate cannot be rejected for both roll and yaw attitude. However, in this case, where we use the CMG momentum dynamics represented as Eqs. 6, it is not evident why a cyclicdisturbance at orbital rate can be rejected for the yaw attiude and not for the roll attitude. In order to prove such inherent characteristics of roll/yaw dynamics, the following CMG momentum dynamics with a proper modification of Eqs. 9 are investigated:

$$
\begin{aligned}
& \dot{h}_{1}=u_{1} \\
& \dot{h}_{3}=u_{3}
\end{aligned}
$$

For this case, the following transfer function matrix can be obtained:

$$
\left[\begin{array}{l}
\theta_{1}(s) \\
\theta_{3}(s)
\end{array}\right]=\frac{1}{\Delta}\left[\begin{array}{rr}
-I_{3} s^{2}\left(s^{2}+n^{2}\right), & \left(I_{2}-I_{1}\right) n s\left(s^{2}+n^{2}\right) \\
n s\left(I_{3}-I_{2}\right)\left[s^{2}+(2 n)^{2}\right], & -s^{2}\left[I_{1} s^{2}+\left(I_{1}+3 I_{2}-3 I_{3}\right) n^{2}\right]
\end{array}\right] .
$$




$$
\begin{aligned}
& {\left[\begin{array}{l}
u_{1}(s) \\
u_{3}(s)
\end{array}\right]+\frac{s^{2}}{\Delta}\left[\begin{array}{rr}
I_{3} s^{2}+\left(I_{2}-I_{1}\right) n^{2}, & \left(I_{1}-I_{2}+I_{3}\right) n \cdot s \\
-\left(I_{1}-I_{2}+I_{3}\right) n s, & I_{1} s^{2}+4\left(I_{2}-I_{3}\right) n^{2}
\end{array}\right]\left[\begin{array}{l}
w_{1}(s) \\
w_{3}(s)
\end{array}\right]} \\
& {\left[\begin{array}{l}
h_{1}(s) \\
h_{3}(s)
\end{array}\right]=\frac{1}{s}\left[\begin{array}{ll}
1, & 0 \\
0, & 1
\end{array}\right]\left[\begin{array}{l}
u_{1}(s) \\
u_{3}(s)
\end{array}\right]}
\end{aligned}
$$

where $\Delta=I_{1} I_{3} s^{2}\left[s^{4}+\left(1+3 k_{1}+k_{1} k_{3}\right) n^{2} s^{2}+4 k_{1} k_{3} n^{4}\right], k_{1}=\left(I_{2}-I_{3}\right) / I_{1} \cdot k_{3}=$ $\left(I_{2}-I_{1}\right) / I_{3}$.

It can be shown that $\pm n j$ are transmission zeros of the transfor function matrix from $\left(u_{1}, u_{3}\right)$ to $\left(\theta_{1}, \theta_{3}\right)$. The zero at $s= \pm n j$ appears in both the $\theta_{1}(s) / u_{1}(s)$ and $\theta_{1}(s) / u_{3}(s)$ transfer functions, but not in yaw attitude channels. Therefore, a cyclic-disturbance rejection at the orbital rate is not possible for roll attitude, while it is possible for yaw attitude. Furthermore, it can be shown that $\pm n j$ are not transmission zeros of the transfer function matrix from $\left(u_{1}, u_{3}\right)$ to $\left(h_{1}, \theta_{3}\right)$. Based on these inherent properties of the roll/yaw dynamics, a cyclic-disturbance rejection for the roll-axis CMG momentum and yaw attitude is considered.

The roll/yaw cyclic-disturbance rejection filters proposed for the space station are:

$$
\begin{aligned}
& \ddot{\alpha}_{1}+(n)^{2} \alpha_{1}=h_{1} \\
& \ddot{\beta}_{1}+(2 n)^{2} \beta_{1}=h_{1} \\
& \ddot{\alpha}_{3}+(n)^{2} \alpha_{3}=\theta_{3} \\
& \ddot{\beta}_{3}+(2 n)^{2} \beta_{3}=\theta_{3}
\end{aligned}
$$

Using a LQR synthesis code, we can determine a $2 \times 16$ gain matrix for the roll/yaw equations augmented by the above filter equations. However, we consider here a decentralized, partial state feedback controller in order to minimize the computer storage requirement for gain scheduling.

A decentralized control logic with cyclic-disturbance accommodation is given by:

$$
\begin{aligned}
u_{1}= & K_{1 P} \theta_{1}+K_{1 D} \omega_{1}+K_{1 H} h_{1}+K_{1 I} \int h_{1} \\
& +K_{1 \alpha} \alpha_{1}+K_{1 \dot{\alpha}} \dot{\alpha_{1}}+K_{1 \beta} \beta_{1}+K_{1 \dot{\beta}} \dot{\beta}_{1} \\
u_{3}= & K_{3 P} \theta_{3}+K_{3 D} \omega_{3}+K_{3 H} h_{3}+K_{3 I} \int h_{3} \\
& +K_{3 \alpha} \alpha_{3}+K_{3 \dot{\alpha}} \dot{\alpha_{3}}+K_{3 \beta} \beta_{3}+K_{3 \dot{\beta}} \dot{\beta}_{3}
\end{aligned}
$$


Proper closed-loop eigenvalues are first selected from LQR design iterations, and the sixteen gains of Eqs. 24 are then determined by using the pole placement algorithm derived in A ppendix. These gains and closed-loop eigenvalues are listed in Tables 3 and 4 , respectively. It is emphasized that the decentralized control is not necessary if a state feedback controller with 32 gains can be easily implemented without any on-board computer storage problem for many different operating conditions. The decentralized controller is proposed here as an additional capability of our control system for the space station.

Bode plots of the closed-loop transfer functions are shown in Fig. 6. Both $h_{1}(s) / \mathrm{w}_{1}(s)$ and $\theta_{3}(s) / \mathrm{w}_{3}(s)$ have zeros at $s= \pm n j$ and $\pm 2 n j$; hence, an asymptotic disturbance rejection for the roll-axis CMIG momentum and yaw attitude is achieved for cyclic disturbances of frequencies $n$ and $2 n$. It can be shown that $\pm n j$ are blocking zeros of the closed-loop transfer function matrix from $\left(w_{1}, w_{3}\right)$ to $\left(h_{1}, \theta_{3}\right)$. Also $\theta_{1}(s) / \mathrm{w}_{1}(s)$ and $h_{3}(s) / \mathrm{w}_{3}(s)$ have zeros near $s= \pm n j$ and $\pm 2 n j$, which results in minimizing the cyclic peak of the roll attitude and yaw-axis CMG momentum. The responses to the roll/yaw disturbances listed in Table 1, with initial conditions of $\theta_{1}(0)=\theta_{3}(0)=1 \mathrm{deg}$ and $\omega_{1}(0)=\omega_{3}(0)=0.001 \mathrm{deg} / \mathrm{sec}$, are shown in Fig. 7 . The roll-axis CMG momentum approaches the zero steady-state value, while roll attitude oscillates at orbital rate. The yaw attitude approches a constant steadystate value, while the yaw-axis $C M G$ momentum oscillates at orbital rate. As a result, the overall attitude and CMG momentum oscillations are minimized. In fact, the responses of the decentralized controller with 16 gains compare quite favorably with those of a full-state feedback controller with 32 gains.

From simulations, it was verified that the overall closed-loop system, with the feedback gains of Table 3 , is stable for $\pm 30 \%$ inertia matrix variations, and that it has more than $\pm 10 \mathrm{~dB}$ and $45^{\circ}$ stability margins for each loop.

\section{Conclusions}

We have presented a new approach to the space station momcntum management and attitude control. The proposed control algorithm provides a proper cyclic-disturbance accommodation. We have shown that either pitch attitude or pitch-axis CMG momentum, depending on the specific mission requirements, can 
be held constant. We have also found some inherent physical property of the coupled roll/yaw dynamics in terms of a transmission zero of a multivariable system. A decentralized, partial state feedback controller which requires minimum computer storage requirement for gain scheduling was also developed.

\section{Appendix: Pole Placement Algorithm for Partial State Feedback}

A pole placement algorithm for the decentralized, partial state feedback control design is presented here. This iterative algorithm for a multi-input system is an extension of the Bass-Gura formula for a single-input system.

Consider a linear, time-invariant system with a single input described by:

$$
\begin{aligned}
\dot{\mathbf{x}} & =\mathrm{Ax}+\mathbf{b} u \\
u & =-\mathbf{k x}
\end{aligned}
$$

where $\mathbf{x}$ is the $n$-dimensional state vector, $u$ is the scalar input, $\mathbf{A}$ is an $n \times n$ matrix, $\mathbf{b}$ is an $n \times 1$ column vector, and $\mathbf{k}$ is a $1 \times n$ row vector.

The Bass-Gura formula for determining the feedback gain, $\mathbf{k}$, of a controllable system is then given by

$$
\mathbf{a}_{c}-\mathbf{a}_{o}=\mathbf{k C T}
$$

where

$$
\begin{aligned}
\Delta(s) & =\text { characteristic polynomial }=s^{n}+a_{1} s^{n-1}+\cdots+a_{n-1} s+a_{n} \\
\mathbf{a}_{o} & =\left[a_{10}, a_{20}, \cdots, a_{n o}\right] \text { for the open-loop system } \\
\mathbf{a}_{c} & =\left[a_{1 c}, a_{2 c}, \cdots, a_{n c}\right] \text { for the closed-loop system } \\
\mathbf{C} & =\text { controllability matrix }=\left[\mathbf{b}, \mathbf{A} \mathbf{b}, \cdots, \mathbf{A}^{n-1} \mathbf{b}\right] \\
\mathbf{T} & =\text { upper-Toeplitz matrix whose first row is }\left[1, a_{1}, \cdots, a_{n-1}\right]
\end{aligned}
$$

Modification can be made to the Bass-Gura formula for a multi-input system described by:

$$
\dot{\mathbf{x}}=\mathbf{A} \mathbf{x}+\mathbf{B} \mathbf{u}
$$




$$
\mathbf{u}=-\mathbf{K x}
$$

where $\mathbf{x}$ is the $n$-dimensional state vector, $\mathbf{u}$ is the $m$-dimensional input rector. A is an $n \times n$ matrix, $\mathbf{B}$ is an $n \times m$ matrix. and $\mathbf{K}$ is a $m \times n$ gain matrix.

Let $\mathbf{b}_{i}$ be the $\mathrm{i}$-th column of $\mathbf{B}$; that is, $\mathbf{B}=\left[\mathbf{b}_{1}, \mathbf{b}_{2}, \cdots, \mathbf{b}_{\mathbf{m}}\right]$. Also let $\mathbf{k}_{\mathbf{i}}$ be the i-th row of $\mathbf{K}$. Defining $\mathbf{A}^{(j)}$ be the closed-loop system matrix at $j$-th iteration. the algorithm can now be described as

$$
\begin{aligned}
\dot{\mathbf{x}} & =\mathbf{A}^{(j)} \mathbf{x}+\sum_{i}^{m} \mathbf{b}_{i} d u_{i}^{(j+1)} \\
u_{i}^{(j)} & =u_{i}^{(j-1)}+d u_{i}^{(j)}
\end{aligned}
$$

where $d u_{i}^{(j+1)}$ is used in the $(j+1)$-th iteration to improve the convergence to the desired eigenvalues. The incremental feedback gain is related to $d u_{i}^{(j)}$ as

$$
\begin{aligned}
d u_{i}^{(j)} & =-d \mathbf{k}_{\mathbf{i}}^{(j)} \mathbf{x} \\
\mathbf{k}_{i}^{(j)} & =\mathbf{k}_{i}^{(j-1)}+d \mathbf{k}_{\mathbf{i}}^{(j)}
\end{aligned}
$$

Srall changes in the feedback gains are related to the small changes in the characteristic polynomial coefficients as

$$
d \mathbf{a}^{(j)}=\left[d \mathbf{k}_{1}^{(j)} \mathbf{C}_{1}^{(j)}+\cdots+d \mathbf{k}_{m}^{(j)} \mathbf{C}_{m}^{(j)}\right] \mathbf{T}^{(j)}
$$

where

$$
\begin{aligned}
& \mathbf{C}_{i}^{(j)}=\left[\mathbf{b}_{i}, \mathbf{A}^{(j)} \mathbf{b}_{i}, \cdots,\left(\mathbf{A}^{(j)}\right)^{n-1} \mathbf{b}_{i}\right] \quad(i=1, \cdots, m) \\
& \mathbf{T}^{(j)}=\text { upper-Toeplitz matrix with the first-row, }\left[1, a_{1}^{(j)}, \cdots, a_{n-1}^{(j)}\right]
\end{aligned}
$$

The above iterative algorithm can be considered as a general form for the pole placement for multi-input systems. For the full-state feedback problem, the choice of the gains are not unique. There are some methods for the unique selection of the gains, such as eigensystem assignment approaches (e.g, see [22-24]). In this paper, we consider the case where the number of total feedback gains are specified by the number of the states. Then a pseudo-controllability matrix is formed from the selected rows of $\mathrm{C}^{(j)}$ matrices by replacing the unselected gain components with 
zeros. This approach is only valid when the system is controllable by the selected gain components.

\section{References}

1. Kennel, H.F., "Angular Momentum Desaturation for Skylab Using Gravity Gradient Torques," NASA TM X-64628, Dec. 1971.

2. Hahn, E., Kaczynski, R., and Barrows, D., "Autonomous Momentum Management for the Space Platform," Paper No. 84-2006, AIAA/AAS Astrodynamics Conference, August 1984.

3. Hopkins, M. and Hahn, E., "Autonomous Momentum Management for the CDG Planar Space Station," Paper No. 85-0031, AIAA 23rd Aerospace Sciences Meeting, January 1985.

4. Hahn, E. and Hopkins, M., "Adaptive Momentum Management for the Dual Keel Space Station," Paper No. 87-2596, AIAA Guidance, Navigation, and Control Conference, August 1987.

5. Bishop, L.R., et al., "Proposed CMG Momentum Management Scheme for Space Station," Paper No. 87-2528, AIA Guidance, Navigation, and Control Conference, August 1987.

6. Woo, H.H. and Morgan, H.D., "Momentum Management Concepts for a Space Station," Paper No. 86-2047, AIAA Guidance, Navigation, and Control Conference, August 1986.

7. Hattis, P.D., "Predictive Momentum Management for the Space Station," Journal of Guidance, Control, and Dynamics, Vol.9, No.4, July-August 1986, pp.454-461.

8. Shain, E.B. and Spector, V.A., "Adaptive Torque Equilibrium Control of the Space Station," Paper No. 85-0028, AIA A 23rd Aerospace Sciences Meeting, January 1985.

9. Miller, P.A., "Torque Equilibrium Control of Low Altitude Spacecraft," AIAA Paper No. 87-2532, AIAA Guidance, Navigation, and Control Conference. August 1987. 
10. Kennel, H.F., "Steering Law for Parallel Mounted Double- Gimbalcd Control Moment Gyros: Revision A." NASA TM-82300, January 1981.

11. Kennel. H.F., "Double-Gimbaled Control Moment Gyro Steering Law $\mathrm{C}_{\mathrm{P}}{ }^{-}$ date," NASA/MISFC Internal Memo (ED12-86-52), May 12, 1086.

12. "Adaptive Rigid Body Control for a Space Station," Ford Aerospace/IVDLD. Final Report to NASA Marshall Space Flight Center. Contract No. NASS. 36422, March 1988.

13. Chu, P., Wie, B.. Gretz, B., and Plescia, C., "Space Station Attitude Control: Modeling and Design," to be presented at the AIAA GN\&C Conference, August 1988.

14. Wie, B. and Plescia, C.T., "Attitude Stabilization of Flexible Spacecraft during Stationkeeping Maneuvers," Journal of Guidance, Control, and Dynamics, Vol.7, No.4, Jul.-Aug. 1985, pp.430-436.

15. Wie, B., Lehner, J.A., and Plescia, C.T., "Roll/Yaw Control of a Flexible Spacecraft Using Skewed Bias Momentum Wheels," Journal of Guidance, Control, and Dynamics, Vol.8, No.4, Jul.-Aug. 1986, pp. 447-453.

16. Wie, B. and Bryson. A.E., "On Multivariable Control Robustness Examples: A Classical Approach," Journal of Guidance, Control, and Dynamics, Vol.10, No.1, Jan.-Feb. 1987, pp.118-120.

17. Wie, B. and Byun, K.W., "A New Concept of Generalized Structural Filtering for Active Vibration Control Synthesis," AIAA Paper No. 87-2456 (to appear in the J. of Guidance, Control, and Dynamics).

18. Bryson, A.E., Control of Spacecraft and Aircraft, Class Note, Stanford University, 1987 (to be published as a textbook).

19. Bryson, A.E. and IVie, B., Synthesis of Control Logic for Spacecraft and Aircraft, Lecture Note, AIAA Two-day Short Course, Monterey, CA., August $15-16,1987$.

20. Chen, C.-T., Linear System Theory and Design, Holt. Rinehart and Winston, 1984, pp.488-506.

21. Siljak, D., Large-Scale Dynamic Systems, North-Holland, New York, 1978. 
22. Kautsky, J. and Nichols, N.K., "Robust Eigenstructure Assignment in State Feedback Control," Numerical Analysis Report NA/2/S3, School of Mathematical Sciences, Flinders U., Australia (also see PC-MATLAB User's Guide for pole placement gain selection).

23. Rew, D.IV., Junkins, J.L., and Juang, J.N., "Robust Eigenstructure Assignment by a Projection Method: Applications Using Multiple Optimization Criteria," Paper presented at AAS/AIAA Astrodynamics Conference. August 10-13, 1987.

24. Juang, J.N., Lim, K.B., and Junkins, J.L., "Robust Eigensystem Assignment for Flexible Structures," Paper presented at AIAA Guidance, Navigation. and Control Conference, August 17-19, 1987.

25. Garber, T.B., "Influence of Constant Disturbing Torque on the Motion of Gravity-Gradient Stabilized Satellites," AIAA Journal, Vol.1, No.4, April 1963, pp.968-969.

26. Hughes, P.C., Spacecraft Attitude Dynamics, John Wiley \& Sons, 1086, pp.352353. 
Table 1. Space station parameters

\begin{tabular}{ccc}
\hline Parameters & $\begin{array}{c}\text { Assembly } \\
\text { Flight\#3 }\end{array}$ & \multicolumn{1}{l}{ Phase 1} \\
\hline \multicolumn{3}{c}{ Inertia $\left(\right.$ slug- $\left.\mathrm{ft}^{2}\right)$} \\
$I_{11}$ & $23.22 \mathrm{E} 6$ & $50.28 \mathrm{E} 6$ \\
$I_{22}$ & $1.30 \mathrm{E} 6$ & $10.80 \mathrm{E} 6$ \\
$I_{33}$ & $23.23 \mathrm{E} 6$ & $58.57 \mathrm{E} 6$ \\
$I_{12}$ & $-0.023 \mathrm{E} 6$ & $-0.39 \mathrm{E} 6$ \\
$I_{13}$ & $0.47 \mathrm{E} 6$ & $\underline{0.16 \mathrm{E} 6}$ \\
$I_{23}$ & $-0.011 \mathrm{E} 6$ & $0.16 \mathrm{E} 6$ \\
Aerodynamic & torque $(\mathrm{ft}-\mathrm{lb})$ for Phase 1 \\
$\mathrm{w}_{1}$ & $1+\sin (n t)+0.5 \sin (2 n t)$ \\
$\mathrm{w}_{2}$ & $4+2 \sin (n t)+0.5 \sin (2 n t)$ \\
$\mathrm{w}_{3}$ & $1+\sin (n t)+0.5 \sin (2 n t)$ \\
\hline
\end{tabular}

Table 2. Controller gains without cyclic-disturbance rejection filter for the Phase I space station

\begin{tabular}{lccc}
\hline Gain & Pitch & Roll & Yaw \\
\hline$K_{P}(\mathrm{ft}-\mathrm{lb} / \mathrm{rad})$ & $2.448 \mathrm{E}+2$ & $1.113 \mathrm{E}+3$ & $1.173 \mathrm{E}+3$ \\
$K_{D}(\mathrm{ft}-\mathrm{lb} / \mathrm{rad} / \mathrm{sec})$ & $1.465 \mathrm{E}+5$ & $5.161 \mathrm{E}+5$ & $1.283 \mathrm{E}+6$ \\
$K_{H}(\mathrm{ft}-\mathrm{lb} / \mathrm{ft}-\mathrm{lb}-\mathrm{sec})$ & $7.523 \mathrm{E}-3$ & $5.935 \mathrm{E}-3$ & $1.540 \mathrm{E}-2$ \\
$K_{I}\left(\mathrm{ft}-\mathrm{lb} / \mathrm{ft}-\mathrm{lb}-\mathrm{sec}^{2}\right)$ & $3.546 \mathrm{E}-6$ & $1.105 \mathrm{E}-5$ & $1.990 \mathrm{E}-6$ \\
\hline
\end{tabular}


Table 3. Controller gains with cyclic-disturbance rejection filter for the Phase I space station

\begin{tabular}{lcrc}
\hline Gain & Pitch & \multicolumn{1}{c}{ Roll } & Yaw \\
\hline$K_{P}(\mathrm{ft}-\mathrm{lb} / \mathrm{rad})$ & $3.425 \mathrm{E}+2$ & $7.026 \mathrm{E}+2$ & $9.254 \mathrm{E}+2$ \\
$K_{D}(\mathrm{ft}-\mathrm{lb} / \mathrm{rad} / \mathrm{sec})$ & $1.972 \mathrm{E}+5$ & $4.382 \mathrm{E}+5$ & $4.166 \mathrm{E}+5$ \\
$K_{H}(\mathrm{ft}-\mathrm{lb} / \mathrm{ft}-\mathrm{lb}-\mathrm{sec})$ & $1.089 \mathrm{E}-2$ & $4.779 \mathrm{E}-3$ & $1.817 \mathrm{E}-4$ \\
$K_{I}\left(\mathrm{ft}-\mathrm{lb} / \mathrm{ft}-\mathrm{lb}-\mathrm{sec}^{2}\right)$ & $3.953 \mathrm{E}-6$ & $-8.170 \mathrm{E}-7$ & $-1.469 \mathrm{E}-6$ \\
$K_{\alpha}(\mathrm{ft}-\mathrm{lb} / \mathrm{rad})$ & $1.006 \mathrm{E}-4$ & $4.383 \mathrm{E}-10^{*}$ & $-3.375 \mathrm{E}-4$ \\
$K_{\dot{\alpha}}(\mathrm{ft}-\mathrm{lb} / \mathrm{rad} / \mathrm{sec})$ & $5.659 \mathrm{E}-2$ & $7.904 \mathrm{E}-7^{*}$ & $6.606 \mathrm{E}-3$ \\
$K_{\beta}(\mathrm{ft}-\mathrm{lb} / \mathrm{rad})$ & $1.434 \mathrm{E}-5$ & $-3.309 \mathrm{E}-9^{*}$ & $-4.503 \mathrm{E}-4$ \\
$K_{\dot{\beta}}(\mathrm{ft}-\mathrm{lb} / \mathrm{rad} / \mathrm{sec})$ & $7.608 \mathrm{E}-2$ & $-4.940 \mathrm{E}-7^{*}$ & $2.412 \mathrm{E}-1$ \\
\hline
\end{tabular}

* in units of ft-lb/ft-lb-sec ${ }^{3}, \mathrm{ft}-\mathrm{lb} / \mathrm{ft}-\mathrm{lb}-\mathrm{sec}^{2}, \mathrm{ft}-\mathrm{lb} / \mathrm{ft}-\mathrm{lb}-\mathrm{sec}^{3}$, and $\mathrm{ft}-\mathrm{lb} / \mathrm{ft}-\mathrm{lb}-\mathrm{sec}^{2}$, respectively.

Table 4. Closed-loop eigenvalues for the Phase I in units of orbital rate, $n=0.0011 \mathrm{rad} / \mathrm{sec}$

\begin{tabular}{l|cc|cc}
\hline & \multicolumn{2}{|c|}{ Momentum/Attitude } & \multicolumn{2}{c}{ Disturbance Filter } \\
\hline Pitch & $-1.0,-1.5$ & $-1.5 \pm 1.5 j$ & $-0.3 \pm 1.0 j$ & $-0.3 \pm 2.0 j$ \\
\hline \multirow{2}{*}{ Roll/Yaw } & $-0.23,-0.68$ & $-0.66 \pm 1.51 j$ & $-0.23 \pm 0.92 j$ & $-0.20 \pm 2.02 j$ \\
& $-1.02 \pm 0.29 j$ & $-1.50 \pm 0.84 j$ & $-0.26 \pm 1.04 j$ & $-0.62 \pm 2.29 j$ \\
\hline
\end{tabular}


Figure Captions

Figuer 1 The Phase I space Station

Figure 2 The pitch-axis momentum/attitude control system

Figure 3 Pitch-axis response without disturbance rejection filtering Figure 4 Pitch-axis response with disturbance rejection filtering
for the pitch attitude

Figure 5 Pitch-axis response with disturbance rejection filtering for the pitch-axis CMG momentum

Figure 6 Closed-loop frequency response for the roll/yaw axes with disturbance rejection filtering for the yaw attitude and roll-axis CMG momentum

Figure 7 Roll and yaw responses 


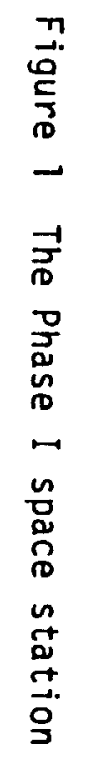

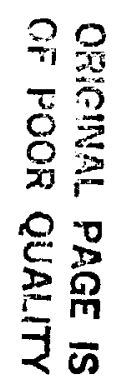

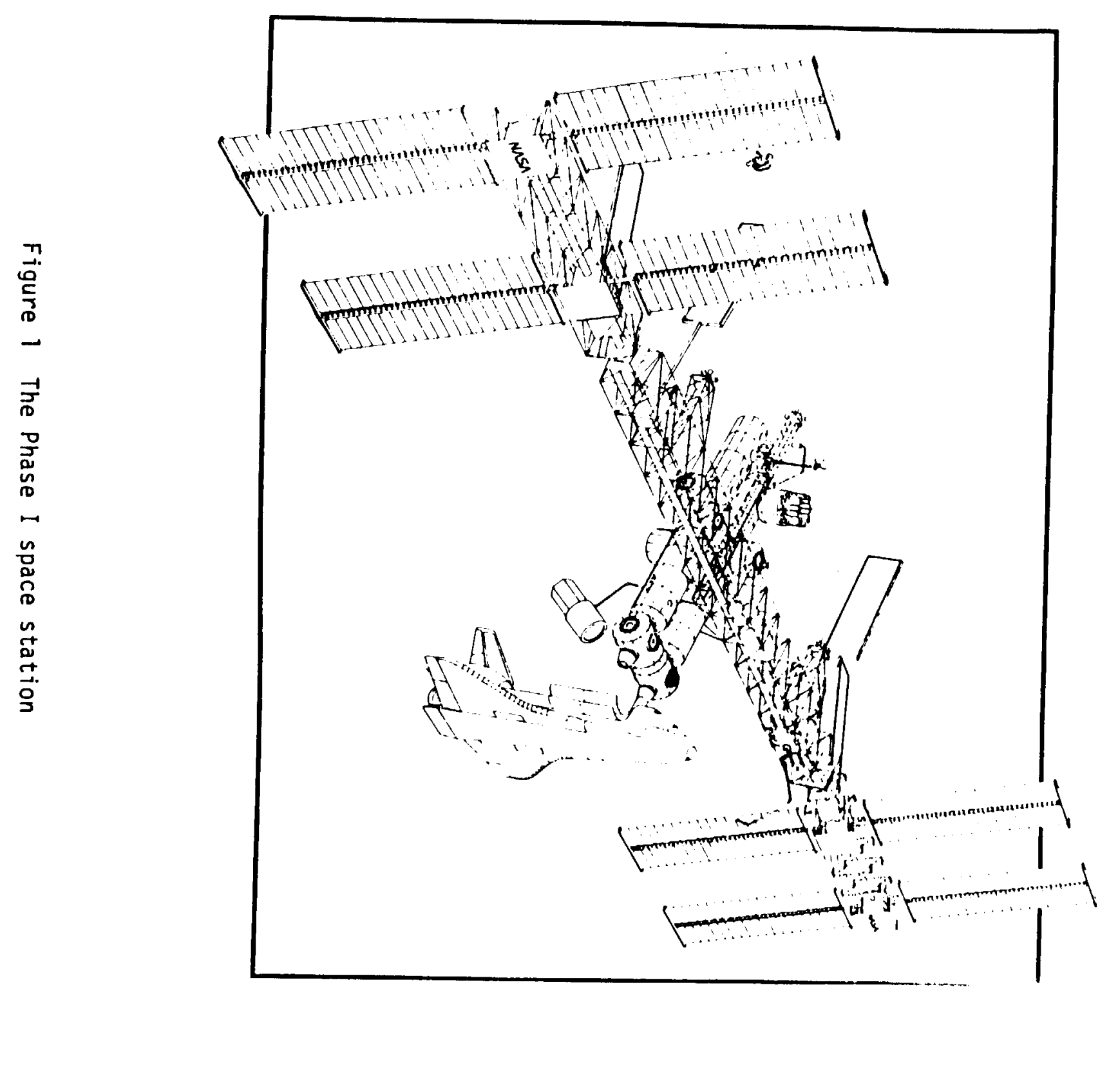




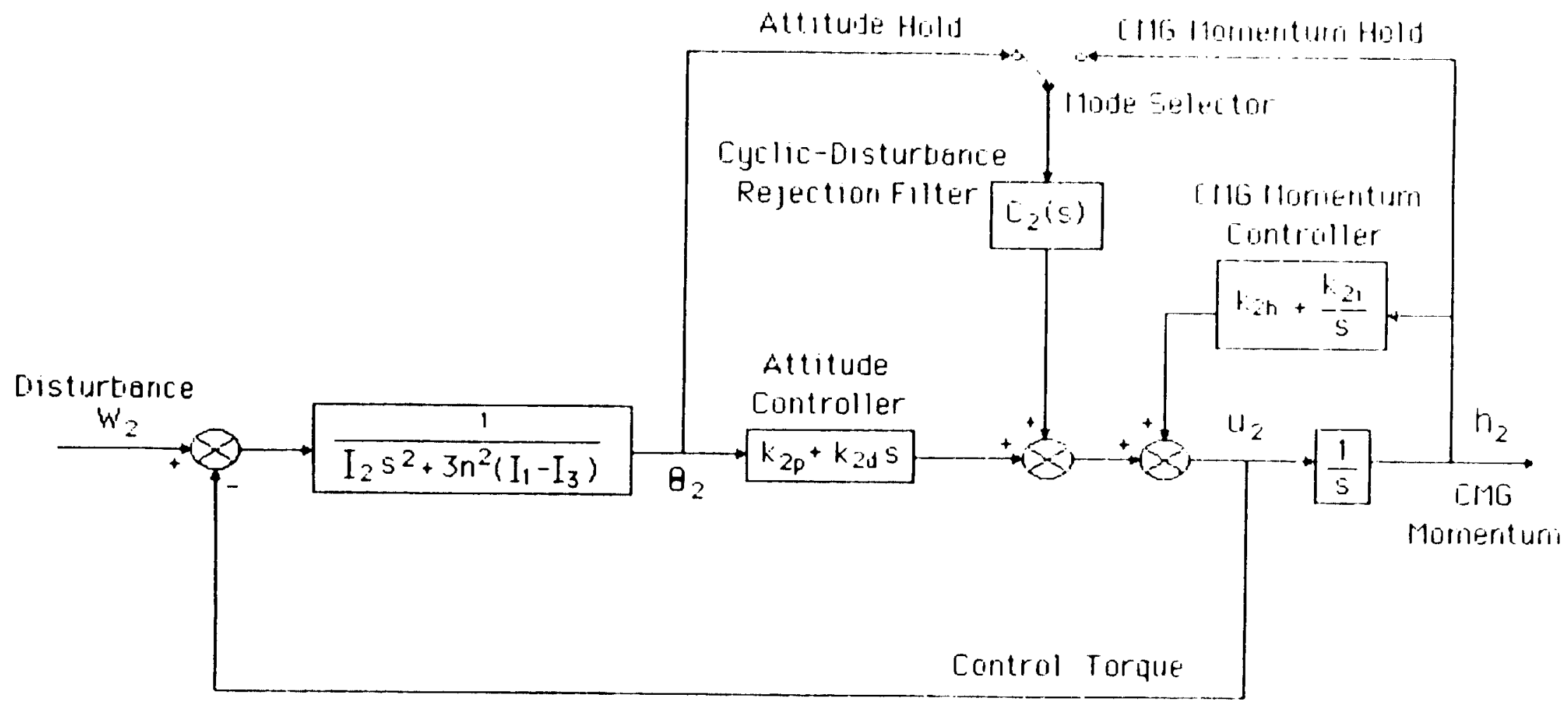

Figure 2 The Pitch-Axis llomenturniAttitude Control System

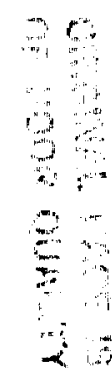




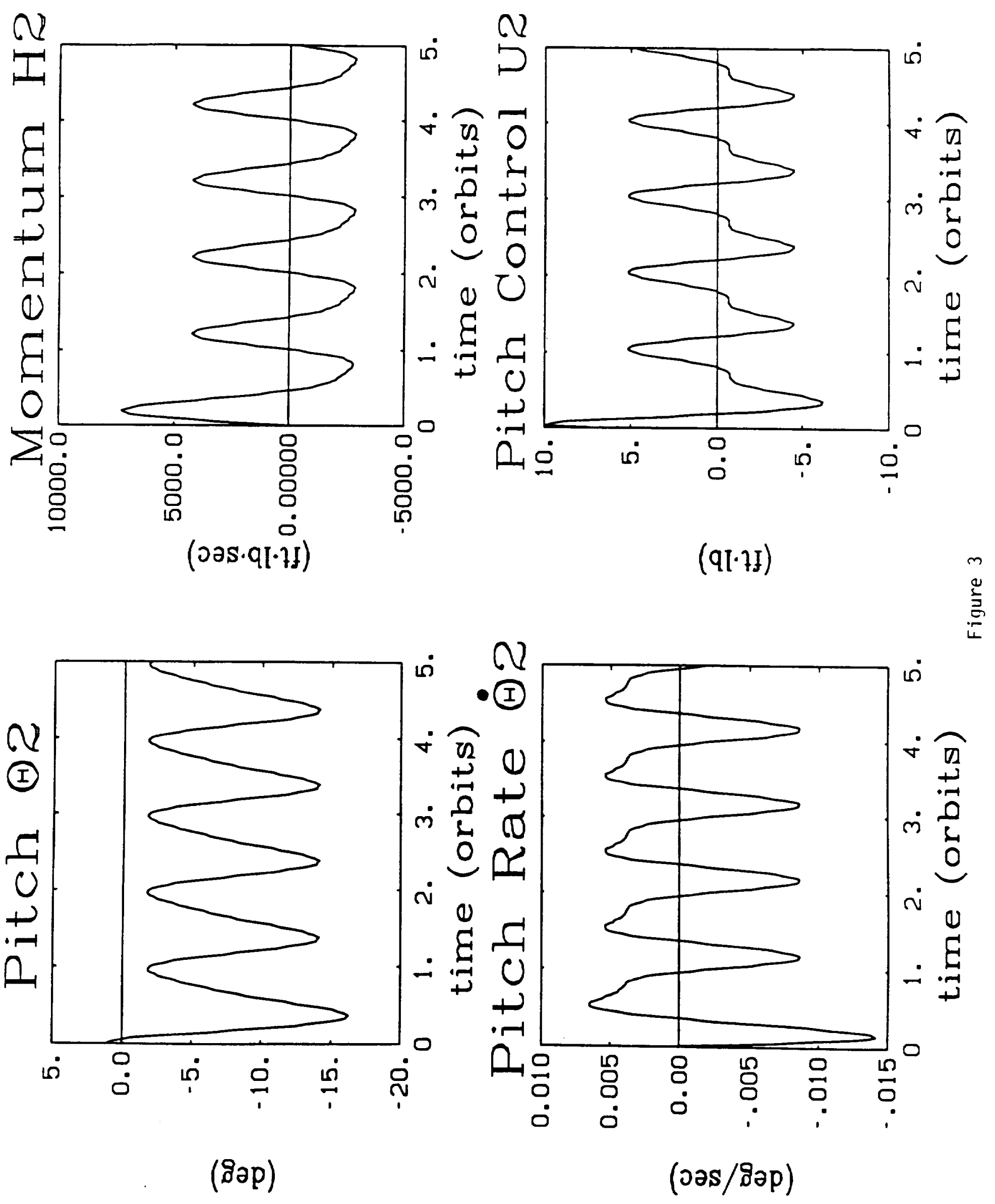



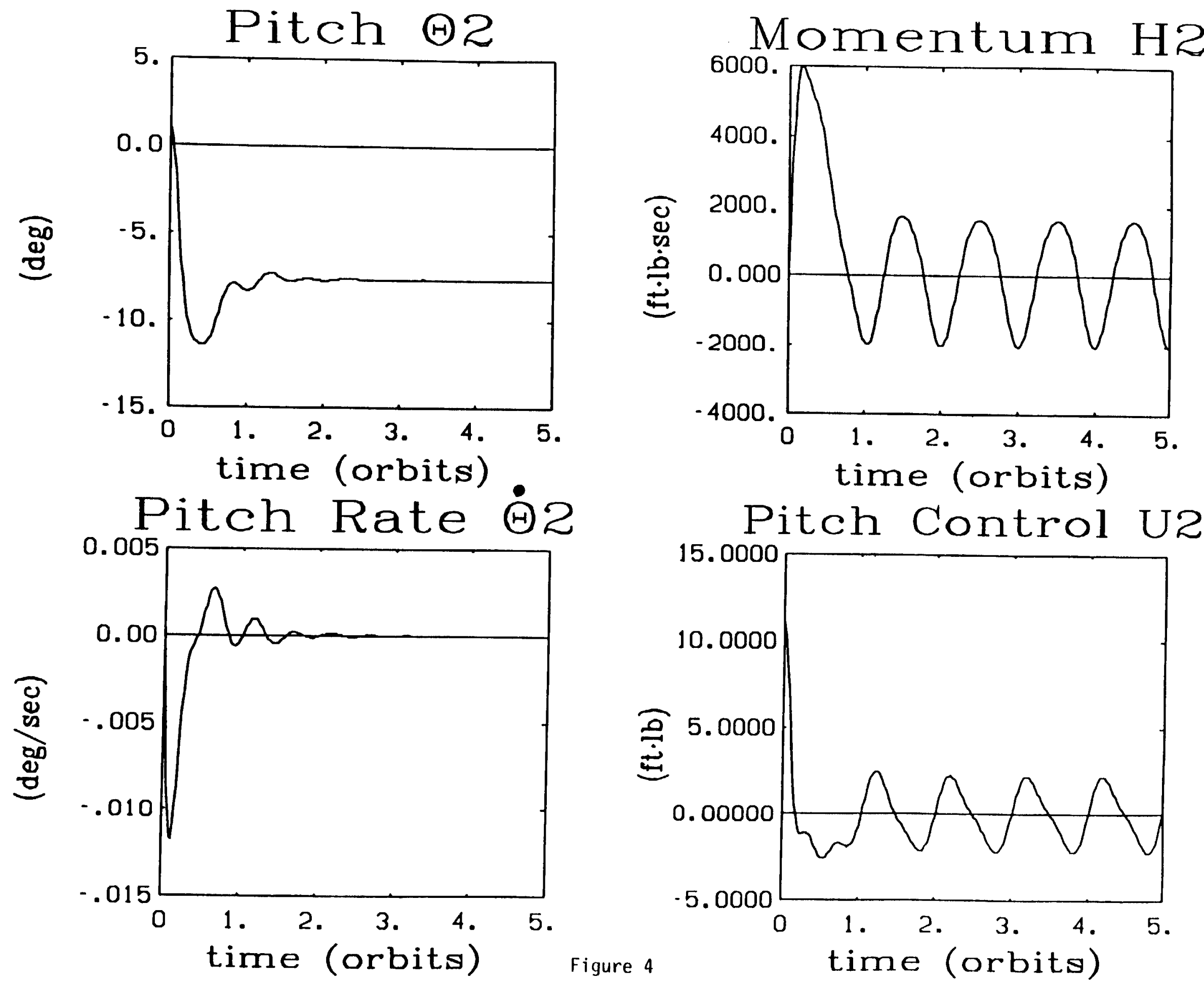

Figure 4 


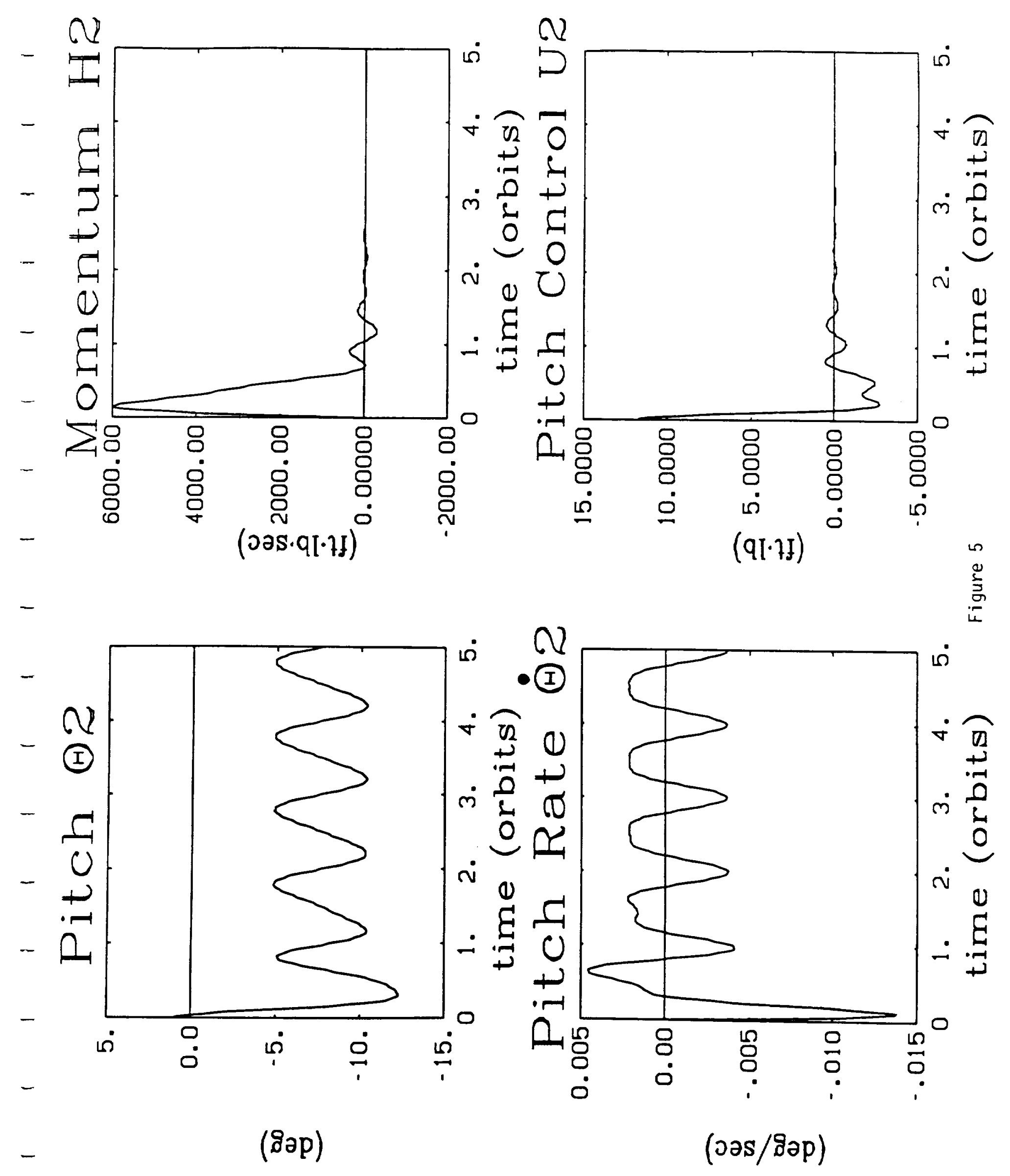



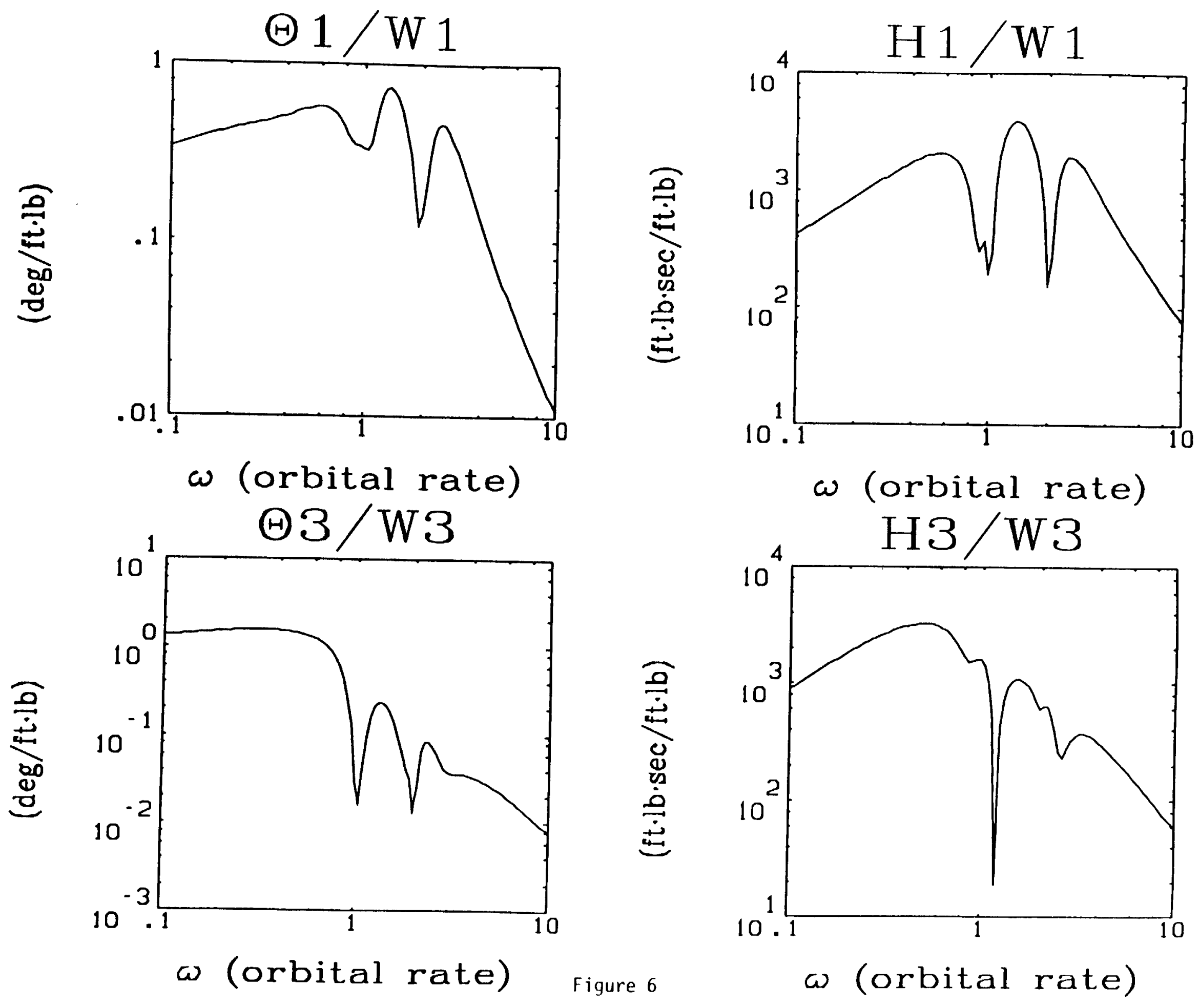

Figure 6 

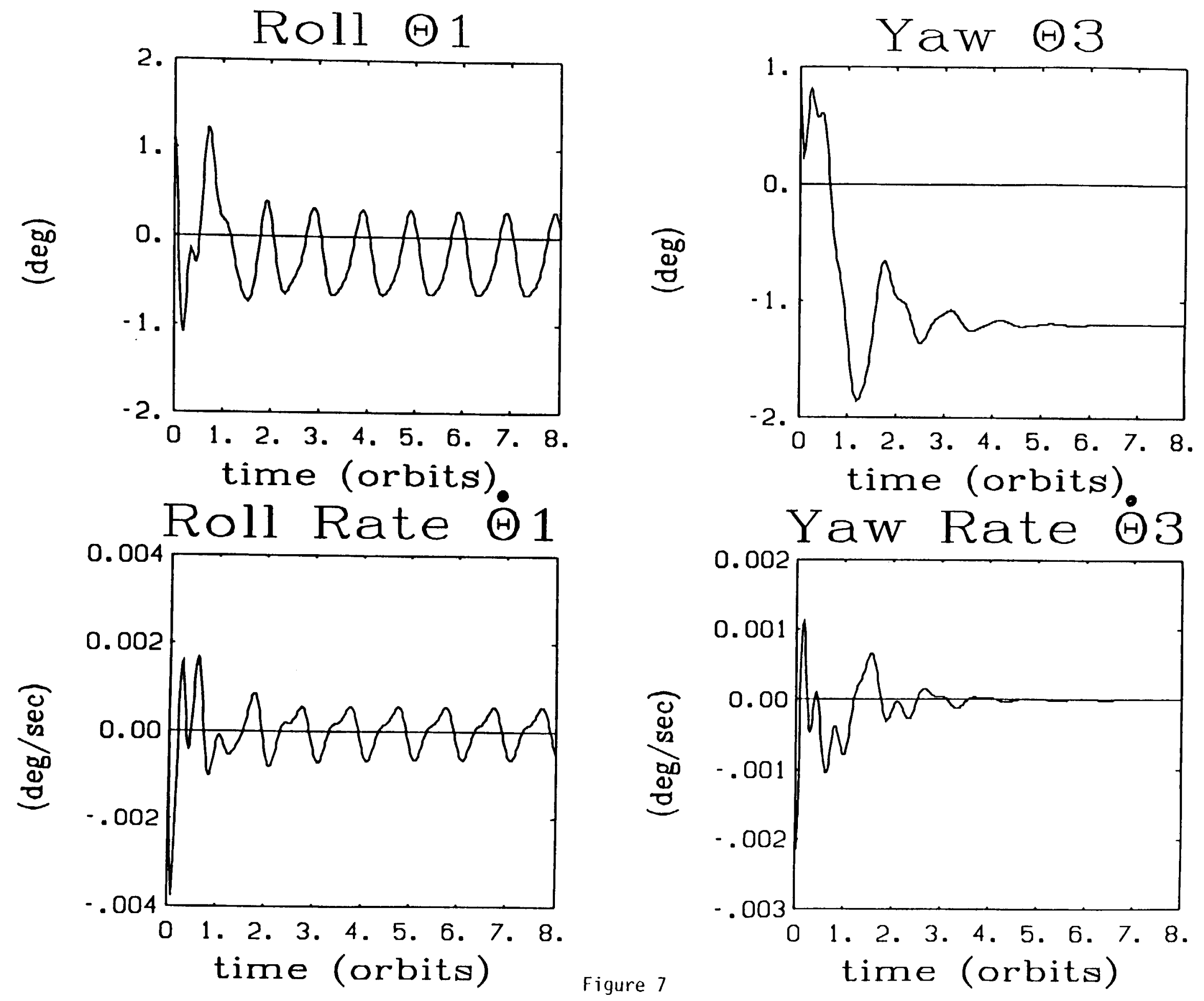

Figure 7

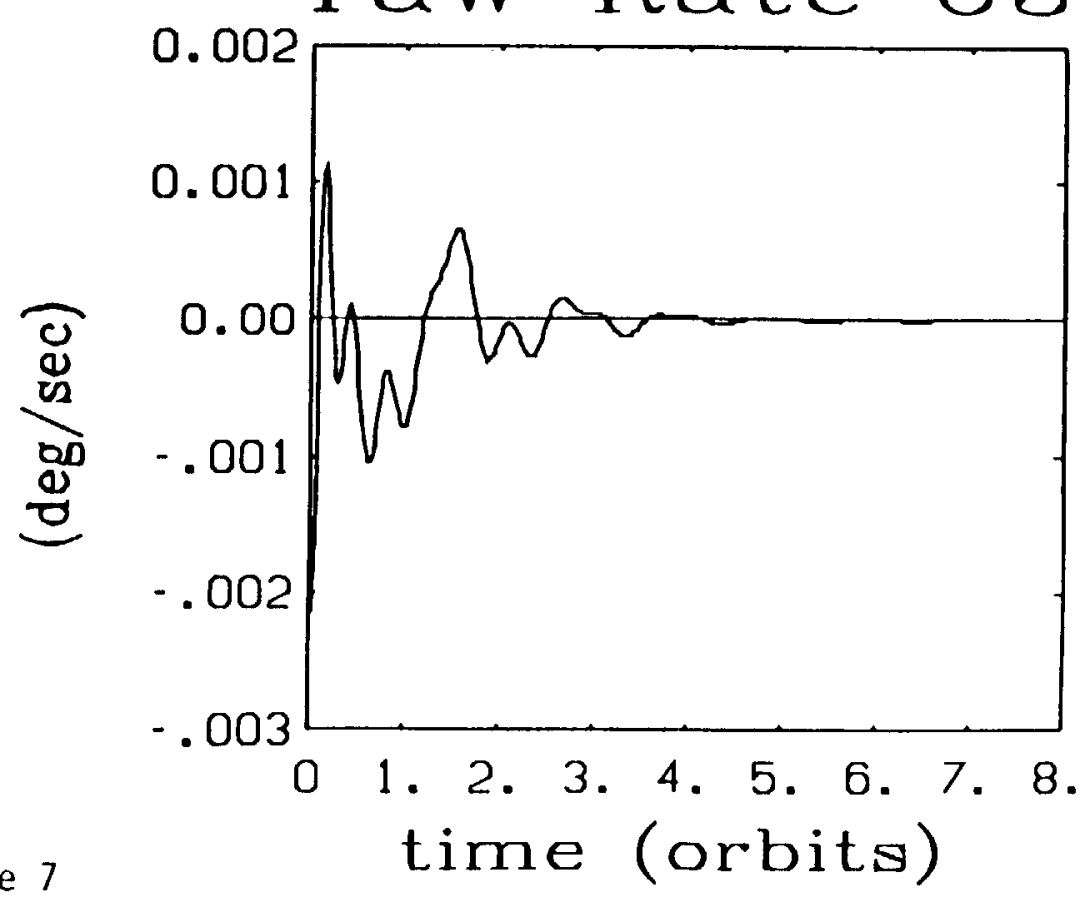



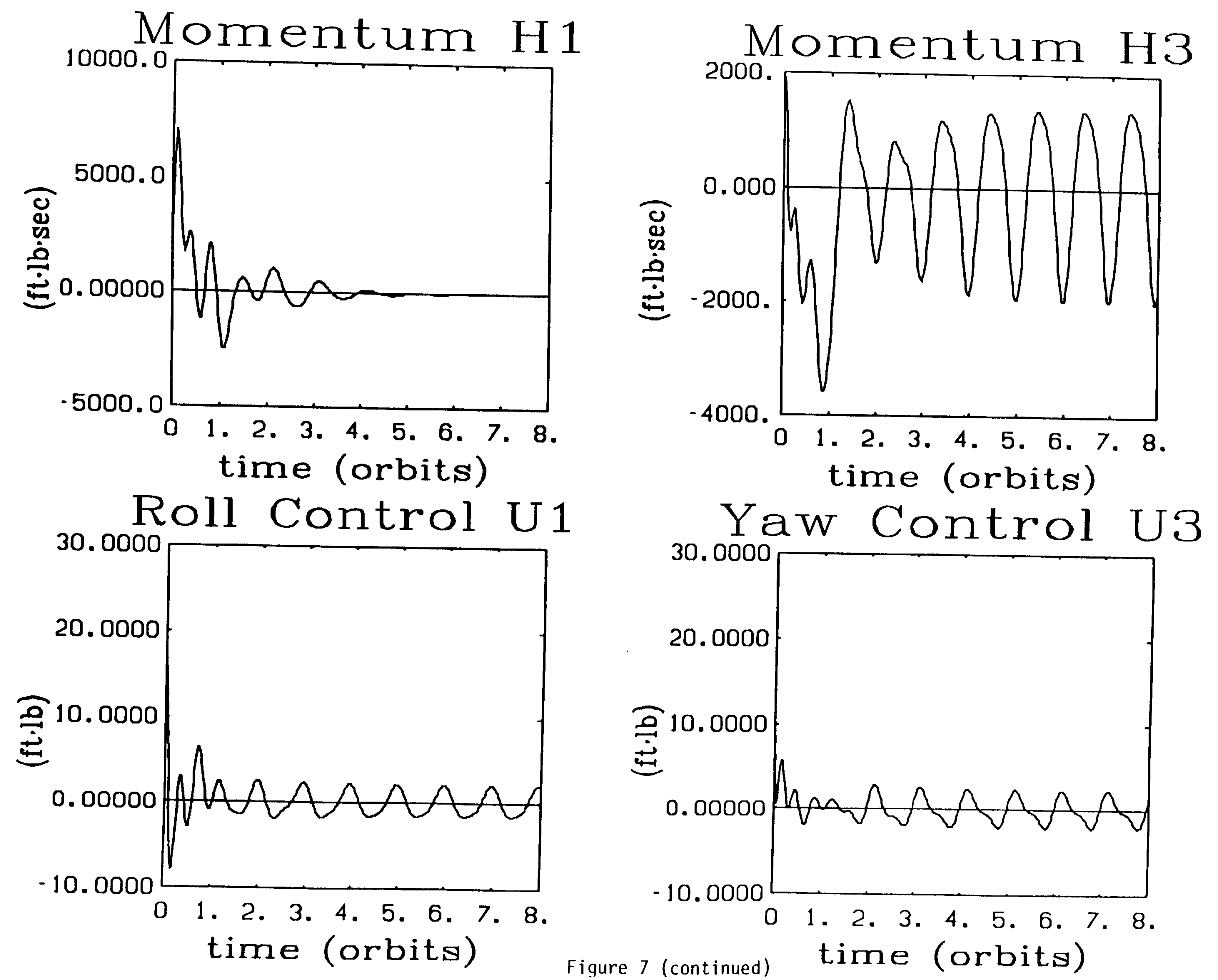

Figure 7 (continued) 
